\title{
Localization of Excitons in the Wetting Layer Accompanying Self-Assembled InAs/GaAs Quantum Dots
}

\author{
A. Babinski ${ }^{a, b}$, S. Raymond ${ }^{c}$, Z. Wasilewski ${ }^{c}$, J. LAPointe $^{c}$ \\ AND M. POTEMSKI ${ }^{a}$ \\ ${ }^{a}$ Grenoble High Magnetic Field Laboratory, MPIFKF and CNRS \\ 38042 Grenoble, France \\ ${ }^{b}$ Institute of Experimental Physics, Warsaw University \\ Hoża 69, 00-681 Warszawa, Poland
}

${ }^{c}$ Institute for Microstructural Sciences, NRC Canada, Ottawa, Canada

\begin{abstract}
A wetting layer is a narrow, highly strained quantum well, which accompanies quantum dots grown in Stranski-Krastanow mode. Its importance for a full description of the quantum dots properties has recently been pointed out. It has been shown for example that excitons can be localized by potential fluctuations in the wetting layer. This is equivalent to the formation of "natural" quantum dots in the WL. Excitonic emission from the single dots formed in the wetting layer accompanying the InAs/GaAs self-assembled quantum dots has been investigated in a high magnetic field (up to $23 \mathrm{~T}$ ). Quadruplet splitting of the investigated emission line has been observed. The attribution of the emission line to the recombination of negatively charged exciton is discussed.
\end{abstract}

PACS numbers: 71.35.Pq, 71.35.Ji, 71.70.Ej

\section{Introduction}

Semiconductor quantum dots (QDs) have attracted considerable attention among researchers [1], this interest being driven by both the fundamental physics and potential for applications. The QDs are sometimes called "artificial atoms" [2], which suggests that the confined states in QDs do not interact with surrounding media. Such a picture, however, has recently been questioned, as experimental evidence for interaction between the self-assembled QDs and the surrounding wetting 
layer (WL) has been presented. The WL is a narrow, highly strained quantum well (QW), which accompanies the QDs grown in Stranski-Krastanow mode [3]. It has been shown that the lateral coupling between the neighboring QDs is mediated by potential fluctuations in the WL [4]. The existence of several resonances in the photoluminescence (PL) excitation of a single QD has also been attributed to "mixed" optical transitions involving carriers from the WL and the QD [5]. Hybridization of the triply-charged excitons in the QDs with Landau-like levels associated with the two-dimensional density of states in the WL has also been observed in magnetic field [6]. These observations point out the importance of the WL for the full description of the QDs physics.

In this communication we report on magnetoluminescence study of the exciton localization in the WL, accompanying the self-assembled InAs/GaAs QDs. We discuss the field-induced splitting of sharp emission lines and we explain the behavior in terms of recombination of negatively charged exciton.

\section{Experimental technique}

A sample with a single layer of InAs QDs was grown on n-doped GaAs substrate by molecular beam epitaxy. The indium flush technique [7] has been applied to limit the height of the dots to $5 \mathrm{~nm}$. Investigated size of the sample was limited using mesa-patterning. The PL measurements were performed at liquid helium temperature in magnetic field up to $23 \mathrm{~T}$ (Faraday configuration) supplied by a resistive magnet at the Grenoble High Magnetic Field Laboratory. Laser excitation ( $\mathrm{Ar}^{+}$laser, $\lambda=514.5 \mathrm{~nm}$ ) was delivered using a single-mode fiber and focused onto the sample with two microlenses. An estimated laser spot size was $20 \mu \mathrm{m}$ and its position over the sample was controlled by Attocube piezo-stages. The PL was collected using a $600 \mu \mathrm{m}$ core diameter multimode fiber placed above the mesa. The PL spectra were dispersed by a $1 \mathrm{~m}$ double grating monochromator and focused onto a CCD.

\section{Results}

It has been found that the lineshape of a WL-related PL strongly depends on the size of investigated mesa. A featureless PL peak due to recombination of carriers in the WL can be seen from mesas larger than $10 \mu \mathrm{m}$, whereas sharp emission lines can be observed from the sub-micron size mesas (see Fig. 1). In this communication we focus our attention on the spectrum shown in the lowermost panel of Fig. 1, however our conclusions can also be applied to other observed features. It has been found that the emission line marked in Fig. 1 with an arrow splits in perpendicular magnetic field into four components (see Fig. 2). Moreover an additional pair of emission lines emerges at higher energy with increasing magnetic field. The energy of observed emission lines relative to their center of gravity is shown in Fig. 3. It has been found that the excitonic effective $g^{*}$-factor of those 


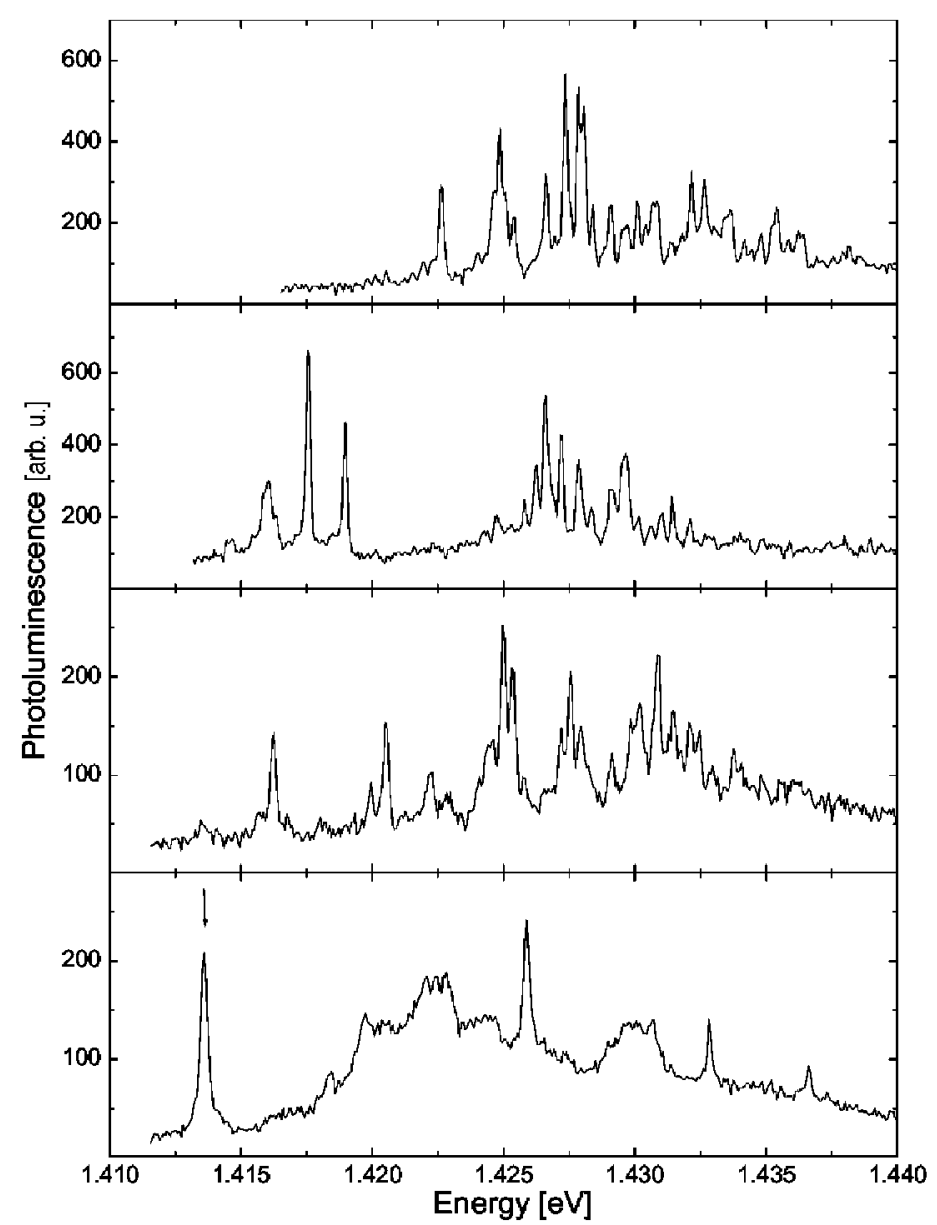

Fig. 1. Photoluminescence spectra from the wetting layer accompanying In As/GaAs self-assembled quantum dots measured on submicron-size mesas at $T=4.2 \mathrm{~K}$ in zero magnetic field. The emission line marked with an arrow in the lowermost panel has been investigated in magnetic field.

three lines is within experimental error the same and equals 1.39 (see the inset to Fig. 3).

\section{Discussion}

The localization of excitons in thin QWs has been studied in several systems i.e. GaAs/AlGaAs [8] or CdTe/CdMgTe [9]. It has also been observed in the WL accompanying the InAlAs/AlGaAs QDs [4]. It is believed that the localization of excitons can be due to monoatomic interface fluctuations. Charged excitons may also be localized by potential fluctuations due to residual charged impurities in the barriers [10]. Finally the 2-3 ML thick bi-dimensional platelets, which form on top of the WL at the early stage of the Stranski-Krastanow transformation may also 


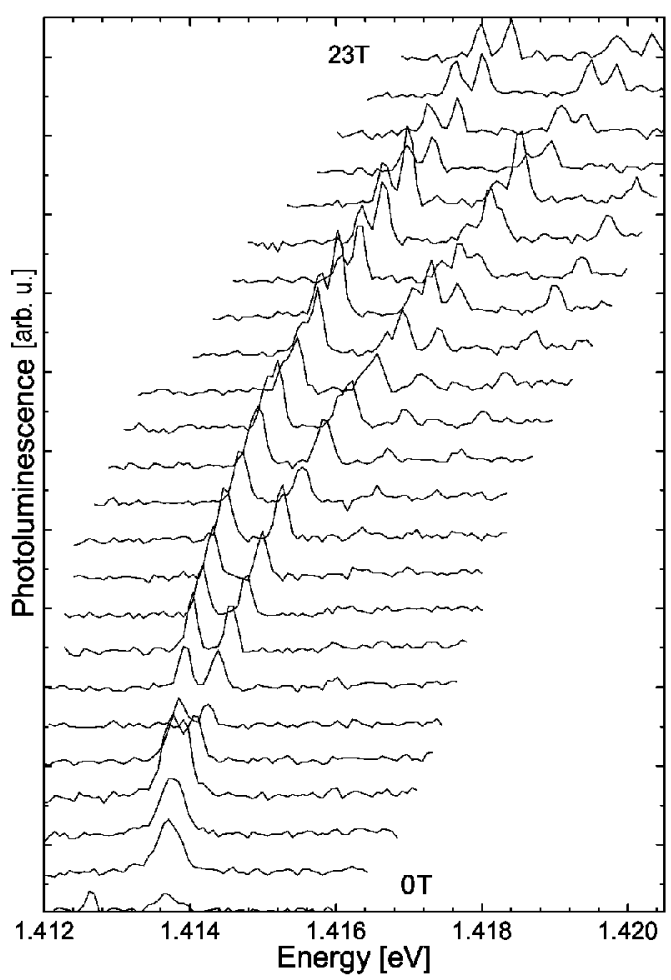

Fig. 2. Evolution of the emission line due to recombination of exciton localized in the wetting layer in magnetic field $(T=4.2 \mathrm{~K})$. Quadruplet splitting and the emerging split-off doublet can be seen.

be responsible for exciton localization [11]. Although no final statement can yet be made on the origin of the investigated localization, the properties of localized excitons can be analyzed.

In the following we focus on the emission line marked with an arrow in Fig. 1. We propose to explain its behavior in magnetic field within the following model. Firstly it should be pointed out that all four split-off lines and very likely the higher-energy doublet appearing at higher magnetic fields have the same excitonic $g^{*}$-factor. It has been found that the $g^{*}$-factors of emission lines from other mesas fluctuate considerably between 1.1 and 1.6. In our opinion this reflects a complicated character of the valence band in the WL, which must be very sensitive to strain fluctuations. The same values of the $g^{*}$-factor observed for emission lines from one submicron-size mesa (see the inset to Fig. 3) strongly suggests their attribution to the same "natural" dot. Our explanation of the observed quadruplet splitting involves a negatively charged exciton in the dot, in which the excess carrier density blocks spin relaxation. Such model has been proposed to explain the quadruplet splitting of emission lines from a single InAs/AlGaAs QD [12] with a zero-field doublet splitting. If the dot is populated by a hole and 


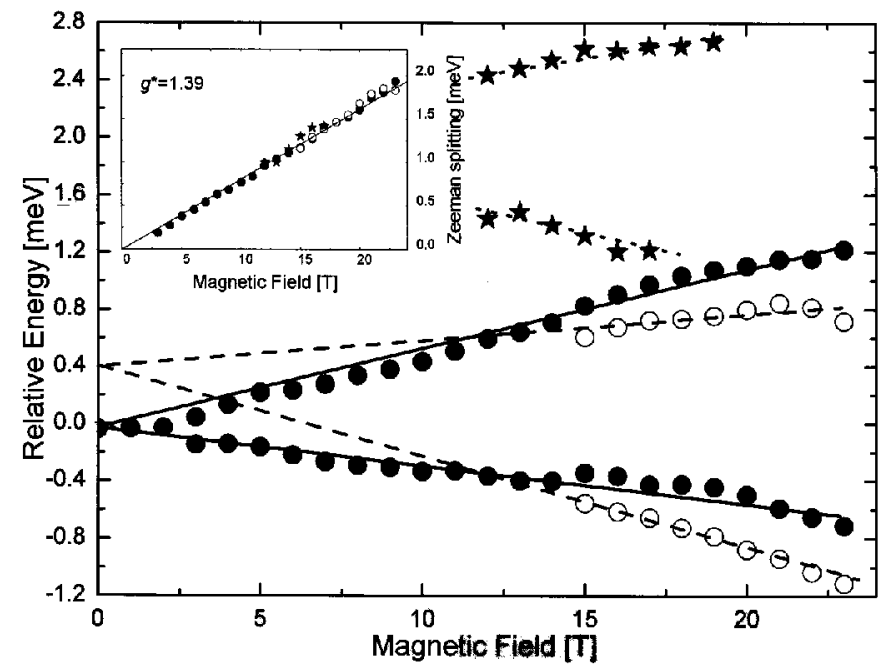

Fig. 3. Recombination energies for the exciton localized in the wetting layer plotted against magnetic field. Diamagnetic shift has been removed for more clarity. The Zeeman splitting of the three pairs of lines is shown in the inset.

two electrons of opposite spin, all carriers can relax to their ground states before recombination. Therefore the recombination of such negatively charged exciton leaves an extra electron in its ground state. However, if the two electrons have the same spins and the spin relaxation is blocked by excess carriers, the exciton recombination takes place leaving the extra electron in the excited state. Although in both cases the excitons recombine from the ground state, their energy differs due to a difference in Coulomb interaction between carriers in those configurations. In the investigated case the spectral width of the emission line $(300 \mu \mathrm{eV})$ at $B=0 \mathrm{~T}$ prevents the observation of its fine structure* ${ }^{*}$ However, the interpolation of the energies of the quadruplet-split lines suggests their zero-field splitting of $400 \mu \mathrm{eV}$ (see the inset to Fig. 3), which is similar to the value observed for the self-assembled InAs/AlGaAs QD [12]. Finally the origin of the spilt-off doublet observed at higher energy (see Fig. 3) can be addressed. In our opinion this must be due to the recombination of a neutral exciton in the same "natural" dot. Non-resonant excitation and relatively long collection time (usually 2 minutes) may favor subsequent charging-discharging processes which should be reflected in the simultaneous observation of both the neutral and the charged exciton. This has previously been observed in self-assembled QDs [13] and quantum rings [14].

*The effect of the emission-line broadening has been observed in the single-dot spectroscopy of self-assembled QDs in mesa-patterned samples. It is very likely that charge fluctuations on lateral surfaces of mesa-structures influence recombination energy of excitons in QDs through the Stark effect. Therefore an emission band rather than a homogeneously broadened line is observed during the CCD measurement (see Ref. [12]). 


\section{Conclusions}

Excitonic emission from the single "natural" dots formed in the WL accompanying the InAs/GaAs self-assembled QDs has been investigated in magnetic field. Quadruplet splitting of the investigated emission line has been observed. The attribution of the emission line to the recombination of negatively charged exciton has been discussed.

\section{Acknowledgments}

A.B. acknowledges financial support from EU under the ICA1-CT- 2002-7009 contract.

\section{References}

[1] L. Jacak, P. Hawrylak, A. Wojs, Quantum Dots, Springer, Berlin 1998; D. Bimberg, M. Grundmann, N.N. Ledentsov, Quantum Dot Heterostructures, John Wiley \& Sons Ltd., New York 1998.

[2] R.C. Ashoori, Nature 379, 413 (1996).

[3] D. Leonard, K. Pond, P.M. Petroff, Phys. Rev. B 50, 11687 (1994).

[4] H.D. Robinson, B.B. Goldberg, J.L. Merz, Phys. Rev. B 64, 075308 (2001).

[5] R. Oulton, J.J. Finley, A.I. Tartakovskii, D.J. Mowbray, M.S. Skolnick, M. Hopkinson, A. Vasanelli, R. Ferreira, G. Bastard, Phys. Rev. B 68, 235301 (2003).

[6] C. Schulhauser, R.J. Warburton, A. Högele, K. Karrai, A.O. Govorov, J.M. Garcia, B.D. Gerardot, P.M. Petroff, Physica E 21, 184 (2004).

[7] Z.R. Wasilewski, S. Fafard, J.P. McCaffrey, J. Cryst. Growth 201/202, 1131 (1999); S. Fafard, Z.R. Wasilewski, C.Ni. Allen, D. Picard, M. Spanner, J.P. McCaffrey, P.G. Piva, Phys. Rev. B 59, 15368 (1999).

[8] D. Gammon, E.S. Snow, B.V. Shanabrook, D.S. Katzer, D. Park, Phys. Rev. Lett. 76, 3005 (1996).

[9] L. Besombes, K. Kheng, D. Martrou, Phys. Rev. Lett. 85, 425 (2000).

[10] G. Finkelstein, H. Shtrikman, I. Bar-Joseph, Phys. Rev. Lett. 74, 976 (1995).

[11] N.P. Kobayashi, T.R. Ramachandran, P. Chen, A. Madhukar,Appl. Phys. Lett. 68, 3299 (1996); K. Yamaguchi, T. Kaizu, K. Yujobo, Y. Saito, J. Cryst. Growth 237-239, 1301 (2002); M. Berti, A.V. Drigo, G. Rossetto, G. Torzo, J. Vac. Sci. Technol. B 15, 1794 (1997); A. Babinski, J. Jasinski, Thin Solid Thins 412, 84 (2002).

[12] M. Bayer, G. Ortner, O. Stern, A. Kuther, A.A. Gorbunov, A. Forchel, P. Hawrylak, S. Fafard, K. Hinzer, J.P. Reithmaier, F. Klopf, F. Schäfer, Phys. Rev. B 65, 195315 (2002).

[13] J.J. Finley, P.W. Fry, A.D. Ashmore, A. Lema ître, A.I. Tartakovskii, R. Oulton, D.J. Mowbray, M.S. Skolnick, M. Hopkinson, P.D. Buckle, P.A. Maksym, Phys. Rev. B 63, 161305 (2001).

[14] R.J. Warburton, C. Schäflein, D. Haft, F. Bickel, A. Lorke, K. Karrai, J.M. Garcia, W. Schoenfeld, P.M. Petroff, Physica E 9, 124 (2001). 